\title{
Simulation of a Waveguide Filter Using Wavelet-Based Numerical Homogenization
}

\author{
Per-Olof Persson ${ }^{*}, 1$ and Olof Runborg $\dagger^{, 2}$ \\ *COMSOL AB, Tegnérgatan 23, SE-111 40 Stockholm, Sweden; and $\dagger$ Program in Applied and Computational \\ Mathematics, Fine Hall, Princeton University, Princeton, New Jersey 08544
}

\begin{abstract}
We apply wavelet-based numerical homogenization to the simulation of an optical waveguide filter. We use the method to derive approximate one-dimensional models and subgrid models of the filter. Numerical examples of the technique are presented, and the computational gains are investigated. (c) 2001 Academic Press

Key Words: wavelets; numerical homogenization; waveguide; filter; subgrid model; multiresolution analysis; Helmholtz equation.
\end{abstract}

\section{INTRODUCTION}

A general problem in the numerical simulation of differential equations is the existence of scales much smaller than the computational domain. Often, small scales in the differential operator or in the solution cannot be resolved by the grid directly because of the overwhelming computational cost this would incur. Discarding the small scales can, however, be tantamount to making large errors. Fine scales in the initial values may for example interact or resonate with fine scales in the material properties and produce coarse-scale contributions to the solution, which would be lost.

A classical analytical way to approximate differential operators containing small scales is homogenization [5]. Simplified homogenized operators can be derived for some classes of small scales and problems, such as linear elliptic equations with periodic or stochastic coefficients. For more complicated problems, however, there is generally no simple way to express the homogenized operator, if it exists.

Recently, the homogenization problem has been approached from a numerical point of view. In [3, 6, 9, 12, 15, 22, 23], it was shown how multiresolution analysis and wavelet

\footnotetext{
${ }^{1}$ The work was carried out at the Department of Numerical Analysis and Computer Science, KTH, Stockholm, Sweden.

${ }^{2}$ The author was partially supported by the NSF KDI grant DMS-9872890. Part of the work was carried out at the Department of Numerical Analysis and Computer Science, KTH, Stockholm, Sweden and at Laboratoire d'Analyse Numérique, Université Paris VI, Paris, France within the EU TMR network H.C.L. ERBFMRXCT960033.
} 


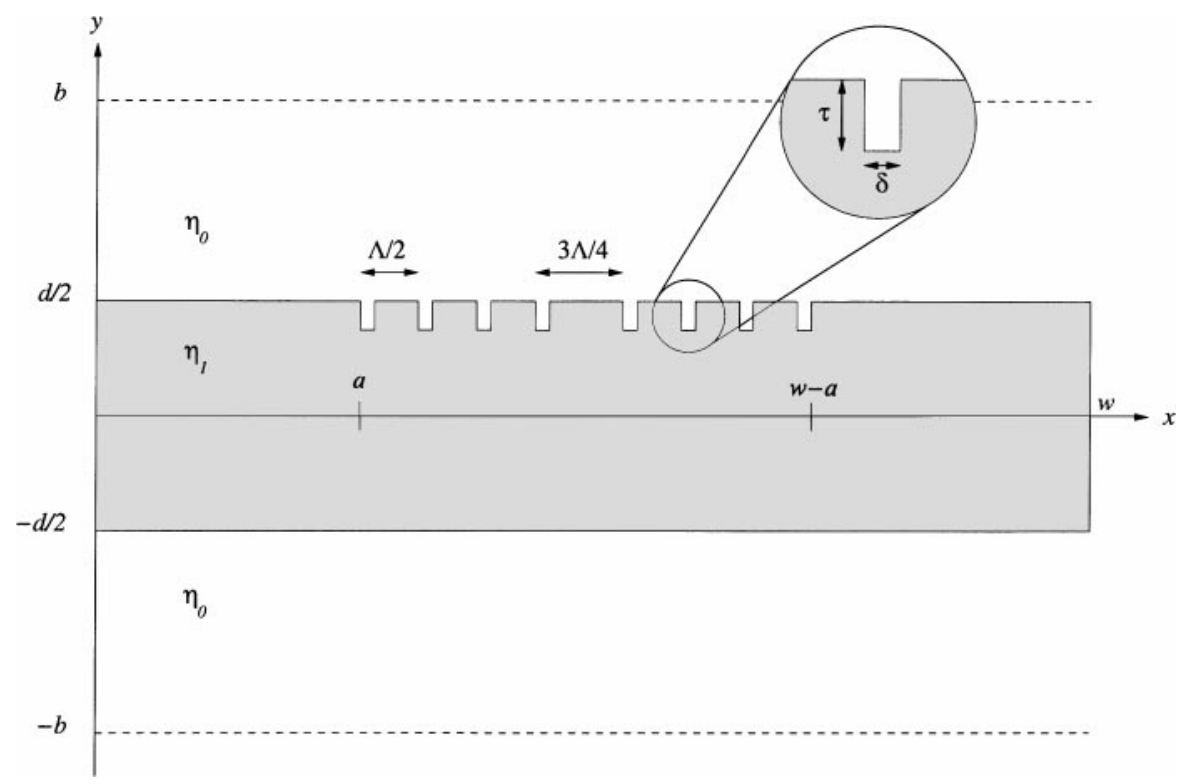

FIG. 1. Specifications of the waveguide under study.

decompositions can be used to derive coarse-scale models to linear partial differential equations. These models can be computed efficiently, and they can be represented in a compact way by sparse matrices. Similar techniques for nonlinear ordinary differential equations have been investigated in [7]. For these methods, which we term numerical homogenization, no assumptions are needed about the structure of the coefficients. In analogy with the analytic case we call the coarse-scale model the (numerically) homogenized operator.

To illustrate the usefulness of wavelet-based numerical homogenization, in this paper we study an optical filter containing a fine-scale structure. The filter is shown in Fig. 1. It is composed of a straight waveguide with small gratings engraved on one of its sides at regular intervals. At one point the distance between two of the grating teeth is increased by $50 \%$. This quarter-wave step gives a narrow-band filter effect and causes waves of one resonant frequency to pass through, while reflecting adjacent frequencies. The ability to separate frequencies makes the filter a useful component in many communication applications. For instance, it allows an optical communication link to be partitioned into many channels where signals can be selectively transmitted and detected. Applications can be found in [17] and [16]. The parameters used in the design of the filter determine many of its relevant properties, and it is of interest to simulate the filter numerically to anticipate the influence of these parameters. The waveguide will be modeled by the two-dimensional Helmholtz equation,

$$
\nabla \cdot\left(c(x, y)^{2} \nabla u\right)+\omega^{2} u=0,
$$

where $c(x, y)$ represents the material-dependent speed of propagation.

The most important feature of the waveguide filter is the interplay between a small-scale periodic structure (the gratings) and a localized inhomogeneity (the quarter-wave step). For this case, analytical homogenization techniques do not apply.

We use wavelet-based numerical homogenization to derive two different simplified models of the filter. First, we show how to compute a one-dimensional model of (1) through 
homogenization only in one coordinate direction. The model is approximately frequencyindependent, and we use it to compute the frequency response of the filter in an efficient manner. Compared to the one computed using the so-called effective index method [4, 20], this response is much more accurate.

Second, we demonstrate how numerical homogenization can be used to compute approximate subgrid models of the different parts of the waveguide. By subgrid model we mean a local discrete model of the effect of scales that the grid does not resolve. We use the subgrid models to solve a large problem on a coarse mesh that does not resolve the gratings. In the first step an operator corresponding to a waveguide with a small number of gratings is homogenized, and the part representing a single grating is identified. The numerically homogenized operator is then extended by replicating this part to obtain the corresponding operator for a waveguide with many gratings. This can be seen as a generalization of solving the cell problem in analytical homogenization and using the computed effective material coefficient in the homogenized equation [5].

Finally, we want to emphasize that neither of the above two methods is in any way constrained to the waveguide problem; they could be used also for other problems where the simplified models are physically feasible.

This paper is organized as follows. In Section 2, we begin by justifying the twodimensional mathematical model used for the optical filter. Next, in Section 3, we show how to approximate and solve those equations with a direct approach. We continue to give a brief introduction to numerical homogenization in Section 4 and finally, in Section 5, we show results of the application of numerical homogenization to the waveguide problem. In particular, we use it to reduce and simplify the direct computational problem, first by a one-dimensional model and second through subgrid modeling as described above.

\section{MATHEMATICAL MODEL}

The Maxwell equations govern the behavior of electromagnetic waves. In our model we will assume that the waves propagate in a medium that is linear, isotropic, nonmagnetic, and nonconducting. The Maxwell equations for the electric field $E=\left(E_{x}, E_{y}, E_{z}\right)$ and the magnetic field $H=\left(H_{x}, H_{y}, H_{z}\right)$ then take the form

$$
\begin{array}{cc}
\mu \frac{\partial H}{\partial t}+\nabla \times E=0, & \epsilon \frac{\partial E}{\partial t}-\nabla \times H=0, \\
\nabla \cdot(\epsilon E)=0, & \nabla \cdot(\mu H)=0,
\end{array}
$$

where $\mu$ and $\epsilon$ represent the medium's permeability and permittivity, respectively. We will only consider waves that are time-harmonic with fixed frequency $\omega$, which amounts to changing $\partial_{t} \mapsto i \omega$ in (2). Taking the time derivative of the first two equations reduces them to

$$
\nabla \times\left(\frac{1}{\mu} \nabla \times E\right)-\epsilon \omega^{2} E=0, \quad \nabla \times\left(\frac{1}{\epsilon} \nabla \times H\right)-\mu \omega^{2} H=0 .
$$

Since the material is nonmagnetic, $\mu$ is constant. We now also assume that $\epsilon$ is constant in the $z$ direction, so that the fields are independent of $z$; hence $\partial_{z}=0$. This gives us two-dimensional Helmholtz equations for the components $E_{z}$ and $H_{z}$,

$$
\nabla^{2} E_{z}+\epsilon(x, y) \mu \omega^{2} E_{z}=0, \quad \nabla \cdot\left(\frac{1}{\epsilon(x, y) \mu} \nabla H_{z}\right)+\omega^{2} H_{z}=0,
$$


where $\nabla$ here and henceforth represents the two-dimensional nabla operator. From $E_{z}$ and $H_{z}$ we can obtain all the remaining components by using (2):

$$
\begin{aligned}
i \omega \mu H_{x} & =\frac{\partial E_{z}}{\partial y}, \quad i \omega \epsilon E_{x}=-\frac{\partial H_{z}}{\partial y}, \\
i \omega \mu H_{y} & =-\frac{\partial E_{z}}{\partial x}, \quad i \omega \epsilon E_{y}=\frac{\partial H_{z}}{\partial x}
\end{aligned}
$$

We will compute a so-called transverse magnetic (TM) mode solution where $E_{z} \equiv 0$. We then only need to find $H_{z}$, which we will denote by $u$ in the remainder of the paper. Let $c_{0}$ be the speed of light in vaccuum. After introducing the relative speed of light $c=c_{0} / \sqrt{\epsilon \mu}$, we finally have the equation for $u$, namely,

$$
\nabla \cdot\left(c(x, y)^{2} \nabla u\right)+\omega^{2} u=0
$$

where for convenience we rescaled $x \mapsto x / c_{0}$ and $y \mapsto y / c_{0}$. In our case $c(x, y)$ is discontinuous (see below) and (6) must be interpreted in the distributional sense. We hence seek a weak solution of (6) in the Sobolev space $H_{\mathrm{loc}}^{1}$. Away from discontinuities in $c$, a weak solution is in fact smooth, satisfying (6) also in the strong sense. Moreover, suppose $c(x, y)$ is discontinuous across a curve in the $x y$ plane with normal $\hat{n}$ and that $u$ is a weak solution of (6). Then it follows that $u$ satisfies the classical interface conditions for Maxwell's equations in lossless media: continuity of $u$ and $c^{2} \hat{n} \cdot \nabla u$ across the curve.

In Fig. 1 we specify the variables that define the dimensions of the waveguide. We will usually fix the length $w=1$ and use $a, d, \Lambda, \delta$, and $\tau$ as design parameters. Related to these is $S$, which will denote the number of gratings. Further parameters are $\eta_{0}$ and $\eta_{1}$, the indices of refraction outside and inside the waveguide, respectively. The index of refraction is defined as $\eta(x, y)=1 / c(x, y)$ and will hence be piecewise constant. Throughout this paper we assume that the material of the waveguide is GaAs, with $\eta=3.3$, and that it is surrounded by vacuum $(\eta=1.0)$.

\section{NUMERICAL APPROXIMATION}

\subsection{Discretization of the Helmholtz Equation}

We start by truncating the physical domain to the rectangle $[0, w] \times[-b, b]$, where $b>d / 2$ is an additional parameter (see Fig. 1). Next, we introduce a uniform grid $\left(x_{k}, y_{\ell}\right)$ of $(N+2) \times(M+2)$ points,

$$
x_{k}=(k-1 / 2) h, \quad y_{\ell}=-b+(\ell-1 / 2) h, \quad h=w / N
$$

for $k=0, \ldots, N+1$ and $\ell=0, \ldots, M+1$. Since we have square cells, the parameters will satisfy the relationship $w M=2 N b$. We will only use $b$ and $d$ values such that $M$ is an integer and $y_{\ell}=d / 2$ for some $\ell$.

Moreover, we define grid functions corresponding to $u(x, y)$ and $c(x, y)$. They are given as

$$
u_{k \ell} \approx u\left(x_{k}, y_{\ell}\right), \quad k=0, \ldots, N+1, \quad \ell=0, \ldots, M+1 .
$$


Letting $x^{+}\left(x^{-}\right)$denote the limiting values from above (below), we get

$$
\begin{array}{lll}
c_{k+1 / 2, \ell}=c\left(x_{k}^{-}+h / 2, y_{\ell}^{*}\right), & k=0, \ldots, N, & \ell=0, \ldots, M+1, \\
c_{k, \ell+1 / 2}=c\left(x_{k}^{-}, y_{\ell}^{*}+h / 2\right), & k=0, \ldots, N+1, & \ell=0, \ldots, M,
\end{array}
$$

where $y_{\ell}^{*}=y_{\ell}^{+}$for positive $y_{\ell}$ and $y_{\ell}^{*}=y_{\ell}^{-}$for negative $y_{\ell}$.

Next, we introduce the difference operators

$$
\begin{array}{ll}
\Delta_{+}^{x} u_{k \ell}=u_{k+1, \ell}-u_{k \ell}, & \Delta_{-}^{x} u_{k \ell}=u_{k \ell}-u_{k-1, \ell}, \\
\Delta_{+}^{y} u_{k \ell}=u_{k, \ell+1}-u_{k \ell}, & \Delta_{-}^{y} u_{k \ell}=u_{k \ell}-u_{k, \ell-1} .
\end{array}
$$

With the above notation we can write down the second-order scheme that we will be using,

$$
\frac{1}{h^{2}} \Delta_{+}^{x} c_{k-1 / 2, \ell}^{2} \Delta_{-}^{x} u_{k \ell}+\frac{1}{h^{2}} \Delta_{+}^{y} c_{k, \ell-1 / 2}^{2} \Delta_{-}^{y} u_{k \ell}+\omega^{2} u_{k \ell}=0
$$

for $k=1, \ldots, N$ and $\ell=1, \ldots, M$. Hence, we use a discretization that corresponds to the same divergence form as the continuous problem (6). This ensures that the numerical solution satisfies the same interface conditions as the weak solution of (6) when $c$ is discontinuous.

\subsection{Boundary Conditions}

To complete the continuous and the discrete problems, (6) and (11), extra conditions must be given at the boundary of the domain $[0, w] \times[-b, b]$. At $y= \pm b$ we use a Neumann boundary condition $u_{y}=0$, discretized with the second-order approximation

$$
\frac{1}{h} \Delta_{+}^{y} u_{k, 0}=0, \quad \frac{1}{h} \Delta_{+}^{y} u_{k, M}=0, \quad k=1, \ldots, N .
$$

This is motivated by the fact that the solution should be almost constant zero far away from the waveguide. Since we are looking for a wave solution propagating along the $x$ axis, we need absorbing boundary conditions at $x=0$ and $x=w$. To derive these boundary conditions we assume that there is a function $f$ and a $\beta \in \mathbb{R}$ such that

$$
u(x \approx 0, y)=f(y) e^{i \beta x}+u_{\text {in }}(y) e^{-i \beta x}, \quad u(x \approx w, y)=\text { const } \cdot f(y) e^{-i \beta x},
$$

where $u_{\text {in }}(y)$ is the amplitude of the injected plane wave. By differentiating (13), we get

$$
u_{x}(0, y)-i \beta u(0, y)=-2 i \beta u_{\text {in }}(y), \quad u_{x}(w, y)+i \beta u(w, y)=0 .
$$

To reduce problems with numerical dispersion and the resulting phase errors, we will choose the discretizations of (14) such that they are exact under the assumption (13). Hence, we want to find $\beta, u_{\text {in }}$, and $a_{j}, j=1,2,3$ such that the grid functions corresponding to (13) satisfy (11) and the boundary conditions

$$
\frac{1}{h} \Delta_{+} u_{0, \ell}+a_{1} u_{0, \ell}=a_{2} u_{\text {in }}\left(y_{\ell}\right), \quad \frac{1}{h} \Delta_{+} u_{N, \ell}+a_{3} u_{N, \ell}=0,
$$

for $\ell=1, \ldots, M$. 
We begin by finding an expression for $\beta$. Close to $x=0$ and $x=w$ the coefficient $c$ is independent of $x$ and we let

$$
c_{\ell}=c\left(y_{\ell}^{*}\right), \quad c_{\ell+1 / 2}=c\left(y_{\ell}^{*}+h / 2\right), \quad \ell \in \mathbb{Z},
$$

where $y_{\ell}^{*}$ is defined as after Eq. (9) and $\left\{y_{\ell}\right\}$ is extended to an infinite grid in the obvious way. For (13) to satisfy (11) the grid function $f_{\ell} \approx f\left(y_{\ell}\right)$ should satisfy the discrete eigenvalue problem

$$
\frac{1}{c_{\ell}^{2} h^{2}} \Delta_{+} c_{\ell-1 / 2}^{2} \Delta_{-} f_{\ell}+\frac{\omega^{2}}{c_{\ell}^{2}} f_{\ell}=\lambda^{2} f_{\ell}, \quad \lambda^{2}=\frac{4}{h^{2}} \sin ^{2} \frac{\beta h}{2}, \quad \ell \in \mathbb{Z} .
$$

The eigenmodes are

$$
\begin{aligned}
f_{\ell} & = \begin{cases}\cos \gamma y_{\ell} & \left|y_{\ell}\right| \leq d / 2, \\
\cos \frac{\gamma d}{2} e^{-\alpha\left(\left|y_{\ell}\right|-d / 2\right)} & \left|y_{\ell}\right|>d / 2,\end{cases} \\
\lambda^{2} & =\eta_{1}^{2} \omega^{2}-\frac{4}{h^{2}} \sin ^{2} \frac{\gamma h}{2}=\eta_{0}^{2} \omega^{2}+\frac{4}{h^{2}} \sinh ^{2} \frac{\alpha h}{2},
\end{aligned}
$$

with $\alpha, \gamma$ given by the transcendental equations

$$
\begin{aligned}
\left(\eta_{1}^{2}-\eta_{0}^{2}\right) \omega^{2} & =\frac{4}{h^{2}}\left(\sin ^{2} \frac{\gamma h}{2}+\sinh ^{2} \frac{\alpha h}{2}\right), \\
e^{\alpha h}-1 & =\frac{\eta_{0}^{2}}{\eta_{1}^{2}}\left(\sin (\gamma h) \tan \left(\frac{\gamma d}{2}\right)-2 \sin ^{2} \frac{\gamma h}{2}\right) .
\end{aligned}
$$

Since we are only interested in the behavior of the lowest propagating mode, we choose the $\beta$ that is related, via the formula in (17), to the largest real eigenvalue $\lambda$. A similar derivation shows that we should use $u_{\text {in }}\left(y_{\ell}\right)=$ const $\cdot f_{\ell}$ as the injected wave at $x=0$, again picking the $f_{\ell}$ that corresponds to the lowest mode.

It remains to find $a_{j}, j=1,2,3$ such that the boundary conditions in (15) are satisfied. By using the simple relation $\Delta_{+} e^{i \beta x_{k}}=\left(e^{i \beta h}-1\right) e^{i \beta x_{k}}$ we immediately get

$$
a_{1}=\frac{1-e^{i \beta h}}{h}, \quad a_{2}=-2 i \frac{\sin (\beta h)}{h} e^{i \frac{\beta h}{2}}, \quad a_{3}=\frac{1-e^{-i \beta h}}{h} .
$$

\section{NUMERICAL HOMOGENIZATION}

In this section we will describe the following general approach to homogenization. Given an equation

$$
L u=f,
$$

where $L$ is a linear operator, $f$ a right-hand side, and $u$ a solution that contains fine scales, find the homogenized operator $\bar{L}$ and right-hand side $\bar{f}$ such that $\bar{L} P u=\bar{f}$, where $P$ is a projection operator onto a subspace in which the fine scales in the original solution do not exist. In our setting (22) will be the finite-dimensional approximation of the Helmholtz equation, described in Section 3, while $P$ will be a projection onto a coarse-scale wavelet space. We will also show how the resulting $\bar{L}$ can be approximated by a sparse matrix. 
Let $H$ be a Hilbert space and $H_{0} \subset H$ a closed subspace, which for our purposes represents the coarse scales of a function space. We denote by $P$ the projection in $H$ onto $H_{0}$ and let $Q=I-P$, where $I$ is the identity operator in $H$. We also introduce the unitary operator $\mathcal{W}$ on $H$ defined by

$$
\mathcal{W}: H \mapsto H_{0} \times H_{0}^{\perp}, \quad \mathcal{W} u=\left(\begin{array}{c}
Q u \\
P u
\end{array}\right) .
$$

For any bounded linear operator $L: H \mapsto H$, we can make the decomposition

$$
\mathcal{W} L \mathcal{W}^{*}\left(\begin{array}{l}
u \\
v
\end{array}\right)=\left(\begin{array}{l}
Q L(Q u+P v) \\
P L(Q u+P v)
\end{array}\right) \equiv\left(\begin{array}{ll}
A & B \\
C & D
\end{array}\right)\left(\begin{array}{l}
u \\
v
\end{array}\right) .
$$

Thus, applying $\mathcal{W}$ to (22) from the left gives

$$
\mathcal{W} L \mathcal{W}^{*} \mathcal{W} u=\mathcal{W} f \Rightarrow\left(\begin{array}{ll}
A & B \\
C & D
\end{array}\right)\left(\begin{array}{l}
Q u \\
P u
\end{array}\right)=\left(\begin{array}{l}
Q f \\
P f
\end{array}\right),
$$

and, assuming that $A$ is an invertible operator, we deduce through block Gaussian elimination that

$$
\bar{L} P u=\bar{f}, \quad \bar{L}=D-C A^{-1} B, \quad \bar{f}=P f-C A^{-1} Q f .
$$

The homogenized operator is thus the Schur complement with respect to the decomposition (24).

We will follow $[3,12]$ and use orthogonal wavelet spaces for the coarse-scale subspace $H_{0}$, as was originally suggested in [6]. If nothing else is stated we have used the Haar basis. For an introduction to wavelets and multiresolution analysis, we refer to [11]. Let $V_{j}$ and $W_{j}$ refer to scaling and wavelet spaces of coarseness level $j$ for some one-dimensional wavelet system. Suppose that $u, f$ in (22) belong to $V_{j+1}$. By definition $V_{j+1}=V_{j} \oplus W_{j}$, and the coarse scale of $u, f$ comprises their projections onto $V_{j}$. We thus let $H=V_{j+1}$ and $H_{0}=V_{j}$ and call the related homogenized operator $\bar{L}_{j}$. If we want to discard more fine scales we can use $H_{0}=V_{j+1-n}$ with $n \geq 1$ and obtain the $n$ levels homogenized operator $\bar{L}_{j+1-n}$. These operators can in fact be obtained through a recursive algorithm, with $\bar{L}_{j-1}$ being the homogenized version of $\bar{L}_{j}$, etc.

In two dimensions we use a standard tensor product extension of the one-dimensional wavelet system, such that $H=V_{j+1} \otimes V_{j+1}$ and $H_{0}=V_{j+1-n_{x}} \otimes V_{j+1-n_{y}}$. The values $n_{x}$ and $n_{y}$ are the number of levels we homogenize in the $x$ and $y$ directions, respectively. This determines how many fine scales to discard. Note that the number of fine scales to discard does not have to be the same in both coordinate directions.

Since we will only work with finite-dimensional problems in this paper we will henceforth discuss the operators in terms of their matrix representations with respect to the wavelet bases. In one dimension the block structure (24) of the linear operator translates to the same block matrix decomposition. In two dimensions we compute $\mathcal{W}$ by taking the Kronecker tensor product with itself of the matrix representation of one-dimensional $\mathcal{W}$. We must thereafter apply a suitable permutation to get the matrix block structure of (24).

It should be noted that in general the matrix $\bar{L}_{j}$ will not be sparse even if $L_{j+1}$ is. For the numerical homogenization to be efficient we must be able to approximate $\bar{L}_{j}$ with a sparse matrix. Theoretical and numerical evidence that this can indeed be done has been given in 
earlier work $[8,9,12]$, where it was also shown that for many practical cases the matrix $A_{j^{\prime}}$ and the resulting $\bar{L}_{j^{\prime}}$ will be strongly diagonal dominant for $j^{\prime} \leq j$. In the present paper we will use a rather simple strategy. In one dimension we approximate $\bar{L}_{j}$ by setting all components outside a prescribed bandwidth $v$ equal to zero. Let us define

$$
\operatorname{trunc}(M, v)_{i j}= \begin{cases}M_{i j}, & \text { if } 2|i-j| \leq v-1, \\ 0, & \text { otherwise. }\end{cases}
$$

This procedure was introduced in [8] and used in [3, 12]. We will use it in this paper to control the structure of $\bar{L}_{j}$ and we refer to it as truncation. A theoretical motivation for this form of approximation is given in [12].

In two dimensions truncation to simple banded form is in general inadequate, since the exact operator will typically be block banded. Let $M$ be the Kronecker tensor product of an $N \times N$ matrix with another matrix. Then we define block banded truncation as

$$
\operatorname{trunc}_{2}(M, v)_{i j}=\left\{\begin{array}{ll}
M_{i j}, & \text { if } 2|i-j-r N| \leq v-1-|2 r|, \\
0, & \text { otherwise, }
\end{array}|2 r|+1 \leq v .\right.
$$

The motivation behind this is the fact that if $L=L_{x} \otimes L_{y}$ then $\bar{L}=\bar{L}_{x} \otimes \bar{L}_{y}$, as shown in [21]. The block banded truncation mimics this, since if $M=M_{v} \otimes M_{u}$ then $\operatorname{trunc}_{2}(M)=$ $\operatorname{trunc}\left(M_{v}\right) \otimes \operatorname{trunc}\left(M_{u}\right)+\delta M$, where the number of nonzero elements in $\delta M$ is very small.

Computing the homogenized operator with a direct method would be expensive. By capitalizing on the nearly sparse structure of the matrices involved, it was however shown in [9] that, for one-dimensional problems, the cost can be reduced to $\mathcal{O}(N)$ operations for $N$ unknowns and fixed accuracy. This method uses a multiresolution-based LU decomposition procedure described in [10]. Moreover, the same homogenized operator will typically be reused multiple times, for instance with different right-hand sides, or in different places of the geometry as a subgrid model. This aspect will be exemplified below in Sections 5.3 and 5.4.

Remark. In the introduction we touched upon analytical homogenization and we would like to compare that with the numerical homogenization procedure described above. The analytical homogenization problem can be formulated as follows. Consider a family of differential operators $\left\{L_{\epsilon}\right\}$ and a right-hand side $f$. Find the homogenized operator $\bar{L}$ such that

$$
L_{\epsilon} u_{\epsilon}=f, \quad \lim _{\epsilon \rightarrow 0} u_{\epsilon}=\bar{u}, \quad \bar{L} \bar{u}=f .
$$

For certain operators the convergence above and existence of the homogenized operator can be proved, for instance in the following $d$-dimensional linear elliptic case [5]. Let $T: \mathbb{R}^{d} \mapsto \mathbb{R}^{d \times d}$ be one-periodic in all coordinate directions, bounded, and uniformly positive definite. If

$$
L_{\epsilon}=-\nabla \cdot T\left(\frac{x}{\epsilon}\right) \nabla
$$

it can be shown that the homogenized operator is given by

$$
\bar{L}=-\nabla \cdot \bar{T} \nabla, \quad \bar{T}=\int_{I_{d}} T(y)-T(y) d \chi(y) d y,
$$

where $I_{d}$ denotes the unit cube in $\mathbb{R}^{d}$ and $d \chi$ is the Jacobian of $\chi: \mathbb{R}^{d} \mapsto \mathbb{R}^{d}$ given as the solution to the so-called cell problem,

$$
\nabla \cdot T(y) d \chi(y)=\nabla \cdot T(y),
$$


with periodic boundary conditions for $\chi$. Note that $\bar{T}$ is a constant matrix. A direct practical application of this can be found in [13].

The structures of the analytical $\bar{L}$ in (31) and the numerical $\bar{L}_{j}$ in (26) are similar. Both are written as the average of the original operator minus a correction term, which is computed in much the same way for both operators. For the analytical case, a local elliptic cell problem is solved to get $T d \chi$, while in the numerical case, a smaller positive definite matrix $A_{j}$ is inverted to obtain $L_{j+1} Q_{j} A_{j}^{-1} B_{j}$. The average over all terms is obtained by integration in the analytical case, and by applying $P_{j}$ in the numerical case. In this sense, computing the numerically homogenized operator is analogous to solving the cell problem in classical homogenization. The relationship can be made more precise in one dimension, where it has been shown that in the limit $\bar{L}_{j}$ approaches $\bar{L}$; see [15, 23].

\section{RESULTS}

\subsection{Simulation}

The left part of Fig. 2 shows examples of solutions in the case of total reflection and total transmission of the injected wave. The specification of the test problem is

$$
\begin{array}{lllll}
w=1, & a=1 / 4, & S=32, & d=17 / 1024, & b=4 / 128, \\
\Lambda=32 / 1024, & \tau=4 / 1024, & \delta=4 / 1024, & M=64, & N=1024,
\end{array}
$$
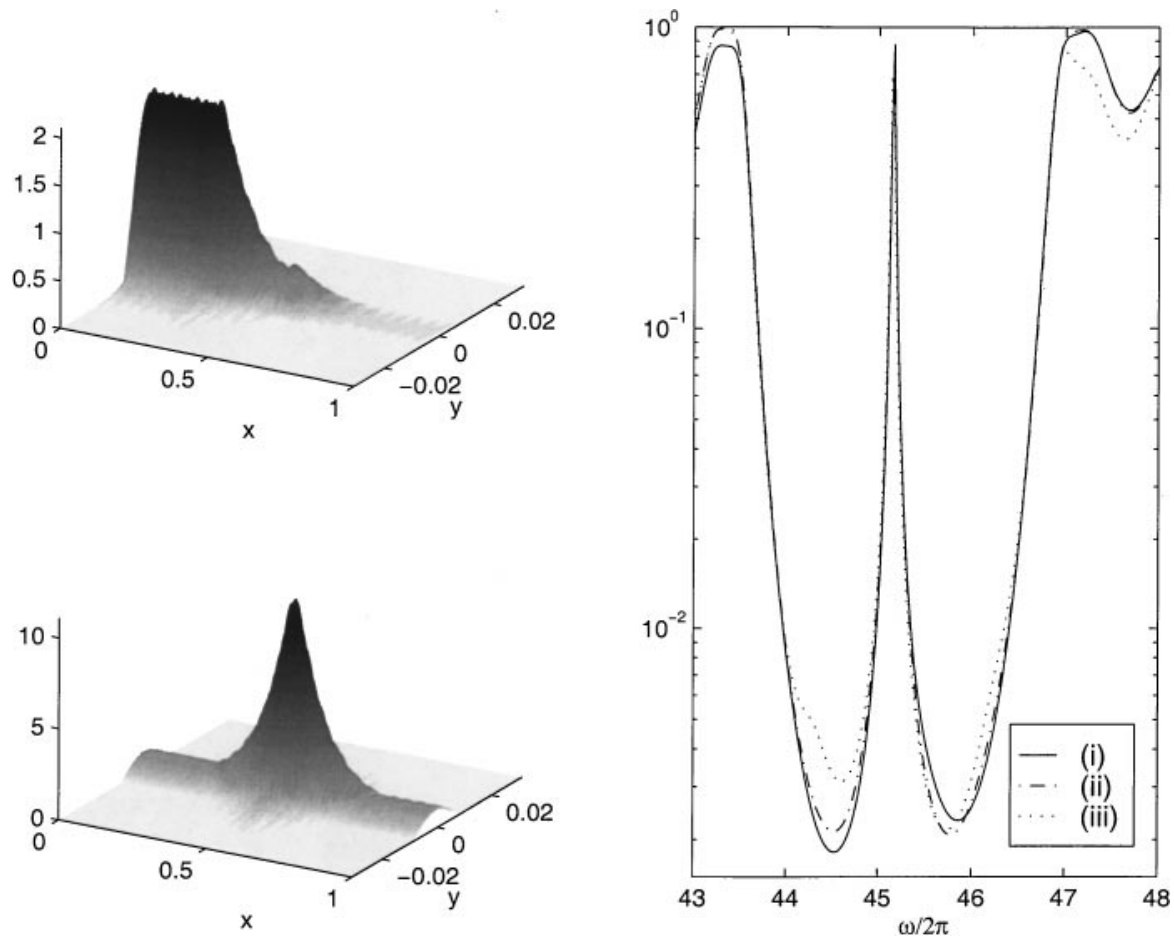

FIG. 2. Plots of solutions to test problem (33). Left figures show $|u|$ for frequencies in the stopband (top left) and at resonance (bottom left). Right figure shows the frequency response $H_{\text {pow }}(\omega)$ for the same test problem (i) and for problems with perturbed $a$ (ii) and $b$ (iii). 
and we used (11) and (21) as the numerical approximation. The two cases were computed with $\omega / 2 \pi=44.5$ (reflection) and $\omega / 2 \pi=45.155$ (transmission).

In the right part of Fig. 2 the frequency response of the same filter (33) is shown. This function measures how much power is transmitted through the filter at each frequency. For a fixed frequency the response is defined and computed as

$$
H_{\text {pow }} \equiv \frac{\int_{-\infty}^{\infty}|u(w, y)|^{2} d y}{\int_{-\infty}^{\infty}\left|u_{\text {in }}(y)\right|^{2} d y} \approx \frac{\sum_{\ell=1}^{M}\left|u_{N, \ell}\right|^{2}}{\sum_{\ell=1}^{M}\left|u_{\text {in }}\left(y_{\ell}\right)\right|^{2}}=\frac{\left\|u_{N, .}\right\|_{2, h}^{2}}{\left\|u_{\text {in }}\right\|_{2, h}^{2}},
$$

where $u_{\text {in }}$ in the last term refers to the grid function $u_{\text {in }}^{\ell} \approx u_{\text {in }}\left(y_{\ell}\right)$. Here we have also introduced the discrete $L_{2}$-norm of a grid function $u_{\ell}$. In one and two dimensions we define it as

$$
\begin{aligned}
& \|u\|_{2, h}=\sqrt{h \sum_{i}\left|u_{i}\right|^{2}} \quad \text { (1D), } \\
& \|u\|_{2, h}=h \sqrt{\sum_{i, j}\left|u_{i j}\right|^{2}} \quad \text { (2D). }
\end{aligned}
$$

The right figure also shows the stability of the response under perturbation of the free variables $a$ and $b$. The case (i) is the original test problem (33). In (ii) we modified $a$ to $a=1 / 8$ (and $N=768, w=3 / 4$ ). In (iii) we changed $b$ to $b=24 / 1024$ (and $M=48$ ). As can be seen, these perturbations do not significantly alter the response.

\subsection{Homogenized Solutions}

The discretizations in Section 3 will lead to a linear system of equations, $L u=f$, where $f$ is a vector determined by the boundary conditions. We apply the technique described in Section 4 to this system and study the result after two levels of homogenization in each coordinate direction.

We will consider the test problem defined by the constants

$$
\begin{aligned}
& w=1, \quad a=3 / 16, \quad S=10, \quad d=9 / 32, \quad b=1 / 8, \\
& \Lambda=1 / 8, \quad \tau=3 / 128, \quad \delta=3 / 128, \quad M=32, \quad N=128 \text {. }
\end{aligned}
$$

In Fig. 3 the solution of this problem at frequency $\omega / 2 \pi=11$ is shown, together with the solution of the homogenized problem. Homogenization was done two levels in both the $x$ and $y$ directions. Note that the homogenized operator was not approximated, and so the homogenized solution is just the projection of the full solution onto the coarse subspace. The effect of approximating the operator using the truncation is shown in Fig. 4. We do both crude truncation according to (27) and block banded truncation according to (28). In the figure we plot the error between the solution obtained using an operator truncated to a varying number of diagnoals and the solution obtained using the full homogenized operator. (To be better able to compare the results we show the number of diagonals, not the $v$ in (27) and (28).) We give relative $L_{2}$ error and relative error in $H_{\text {pow }}$ for both truncation strategies, concluding that the the block truncation method is better, as expected. We also show the strongly diagonal dominant structure of the homogenized operator in Fig. 5. 

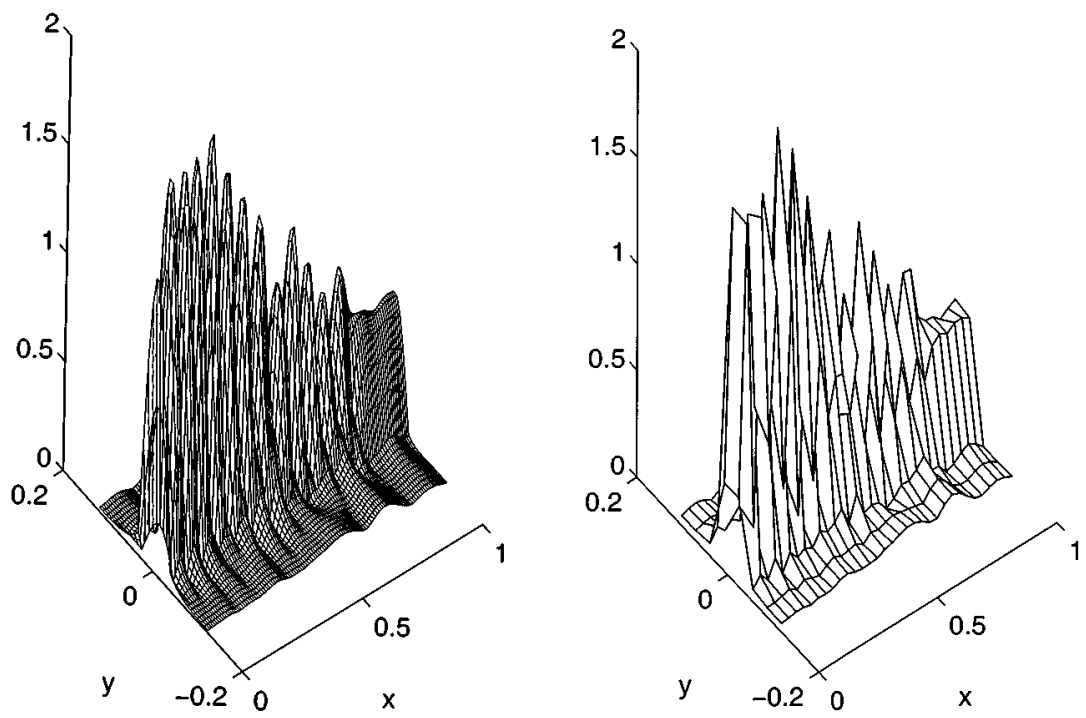

FIG. 3. Solution of test problem (37). Full solution (left) and solution after two levels of homogenization in both $x$ and $y$ directions (right).

Next, we show how to recover the frequency response of the filter (34) from a homogenized solution. Let $P_{j}$ be the projection onto the wavelet space $V_{j}$. In the case of $n_{x}$ and $n_{y}$ levels of homogenization in the $x$ and $y$ directions, we define the homogenized frequency response by simply entering the coarse part of $u$ and $u_{\text {in }}$ (34),

$$
\bar{H}_{\text {pow }}^{n_{x}, n_{y}}=\frac{\left\|\left(\left(P_{j+1-n_{x}} \otimes P_{j+1-n_{y}}\right) u\right)_{\bar{N}, .}\right\|_{2, \bar{h}}^{2},}{\left\|P_{j+1-n_{y}} u_{\text {in }}\right\|_{2, \bar{h}}^{2}},
$$

where $\bar{N}=2^{-n_{x}} N$ and $\bar{h}=2^{n_{y}} h$. In general $\bar{H}_{\text {pow }}^{n_{x}, n_{y}}$ clearly does not agree with $H_{\text {pow }}$. However, in our problem there is a rather simple approximate relationship between the two, which we derive here. Close to $x=w$, the solution should be approximately a right-going
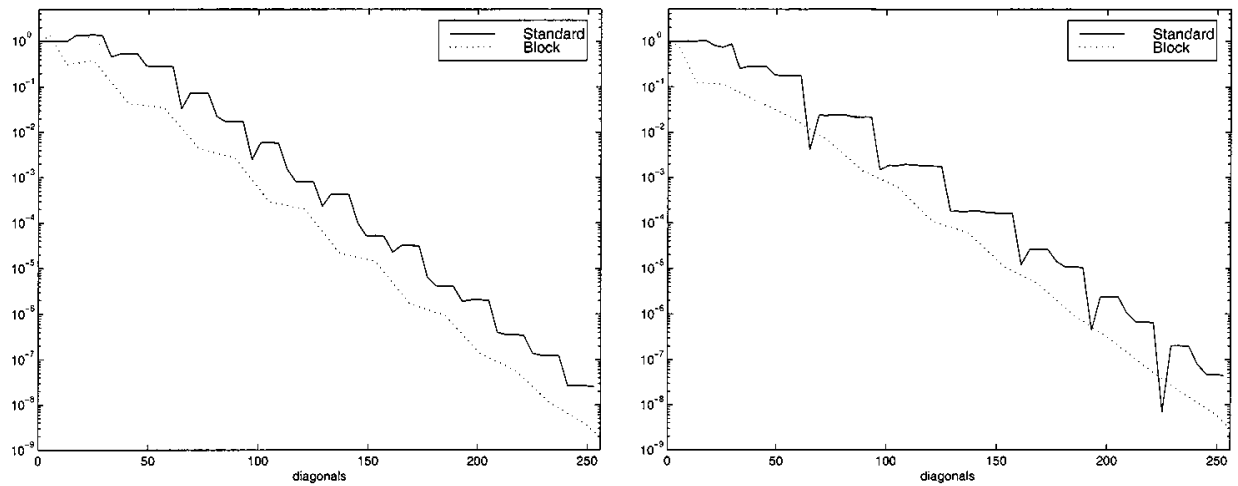

FIG. 4. Relative error of homogenized solution as a function of numbers of diagonals kept, for the two different truncation strategies. Figures show relative $L_{2}$ error (left) and relative $H_{\text {pow }}$ error (right). 


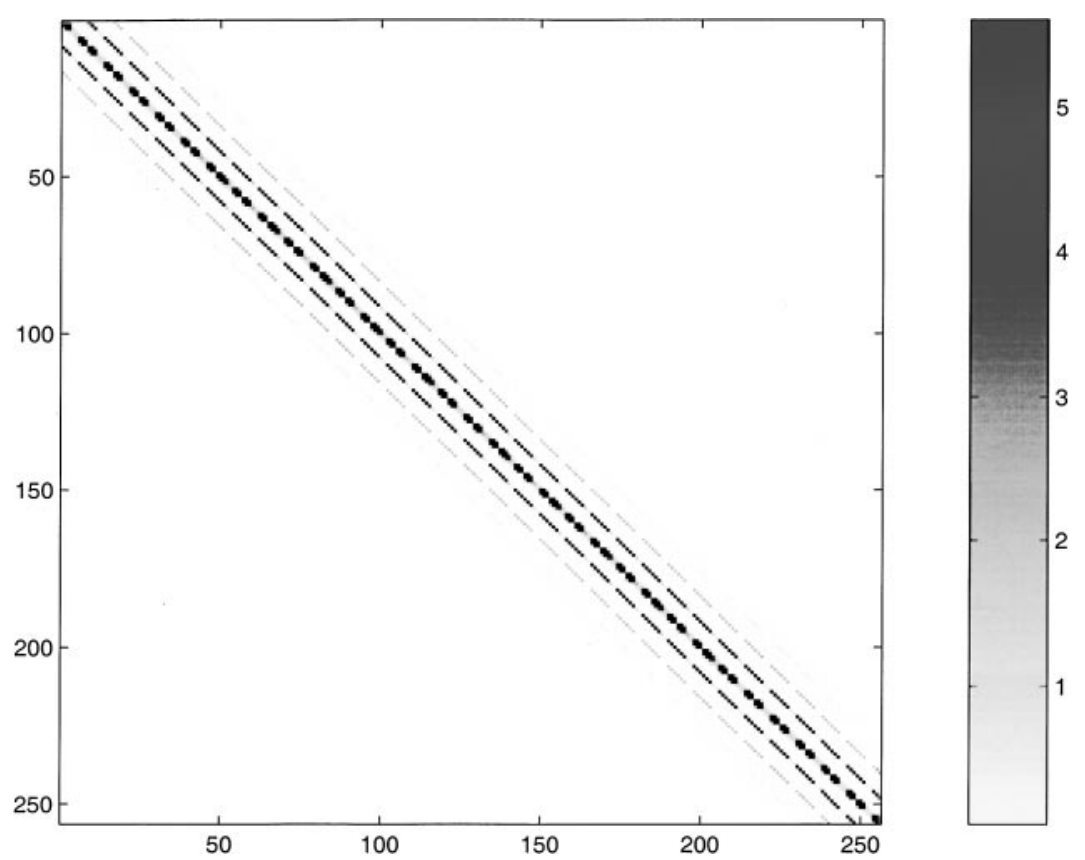

FIG. 5. Structure of the homogenized operator. Absolute values of elements shown.

wave $f(y) e^{-i \beta x}$, with $\beta$ given in Section 3.2. Furthermore,

$$
\left|f\left(y_{\ell}\right)\right| \approx \sqrt{H_{\text {pow }}}\left|u_{\text {in }}\left(y_{\ell}\right)\right|, \quad \forall \ell,
$$

since the shape of the outgoing wave should be the same as the injected wave. This gives us

$$
\begin{aligned}
\bar{H}_{\text {pow }}^{n_{x}, n_{y}} & \approx \frac{\left\|\left(\left(P_{j+1-n_{x}} e^{-i \beta x}\right) \otimes\left(P_{j+1-n_{y}} f\right)\right)_{\bar{N}, .,}\right\|_{2, \bar{h}}^{2}}{\left\|P_{j+1-n_{y}} u_{\text {in }}\right\|_{2, \bar{h}}^{2}} \\
& =\left|\left(P_{j+1-n_{x}} e^{-i \beta x}\right)_{\bar{N}}\right|^{2} \frac{\left\|P_{j+1-n_{y}} f\right\|_{2, \bar{h}}^{2}}{\left\|P_{j+1-n_{y}} u_{\text {in }}\right\|_{2, \bar{h}}^{2}} \approx \alpha_{n_{x}} H_{\text {pow }} .
\end{aligned}
$$

Hence, $H_{\text {pow }}$ is scaled by the frequency-dependent parameter

$$
\alpha_{n_{x}}(\omega)=\left|\left(P_{j+1-n_{x}} e^{-i \beta(\omega) x}\right)_{\bar{N}}\right|^{2},
$$

which is equal to one when $n_{x}=0$. This shows that $H_{\text {pow }} \approx \bar{H}_{\text {pow }}^{n_{x}, n_{y}}$ when we homogenize only in the $y$ direction. In the Haar basis the expression for $\alpha_{n_{x}}$ becomes

$$
\alpha_{n_{x}}=\left|\frac{1}{2^{n_{x}}} \sum_{k=1}^{2^{n_{x}}} e^{-i \beta(w-(k-1) h)}\right|^{2}=\frac{1}{2^{2 n_{x}}}\left|\sum_{k=0}^{2^{n}-1} e^{-i \beta k h}\right|^{2}=\frac{1}{2^{2 n_{x}}}\left|\frac{\sin \left(2^{n_{x}} \beta h\right)}{\sin (\beta h / 2)}\right|^{2},
$$

which is less than or equal to one. For $\beta h \ll 1$ the coefficient $\alpha_{n_{x}} \approx 1-\left(2^{2 n_{x}}-1\right) \beta^{2} h^{2} / 12$.

The frequency response $H_{\text {pow }}$ of the full problem (37) is plotted in Fig. 6. This figure also shows the relative error between $H_{\text {pow }}$ and $\bar{H}_{\text {pow }}^{n_{x}, n_{y}} / \alpha_{n_{x}}(\omega)$ when homogenizing two levels in the $x$ direction, in the $y$ direction, and in both. The error is a few percent, increasing with frequency. 

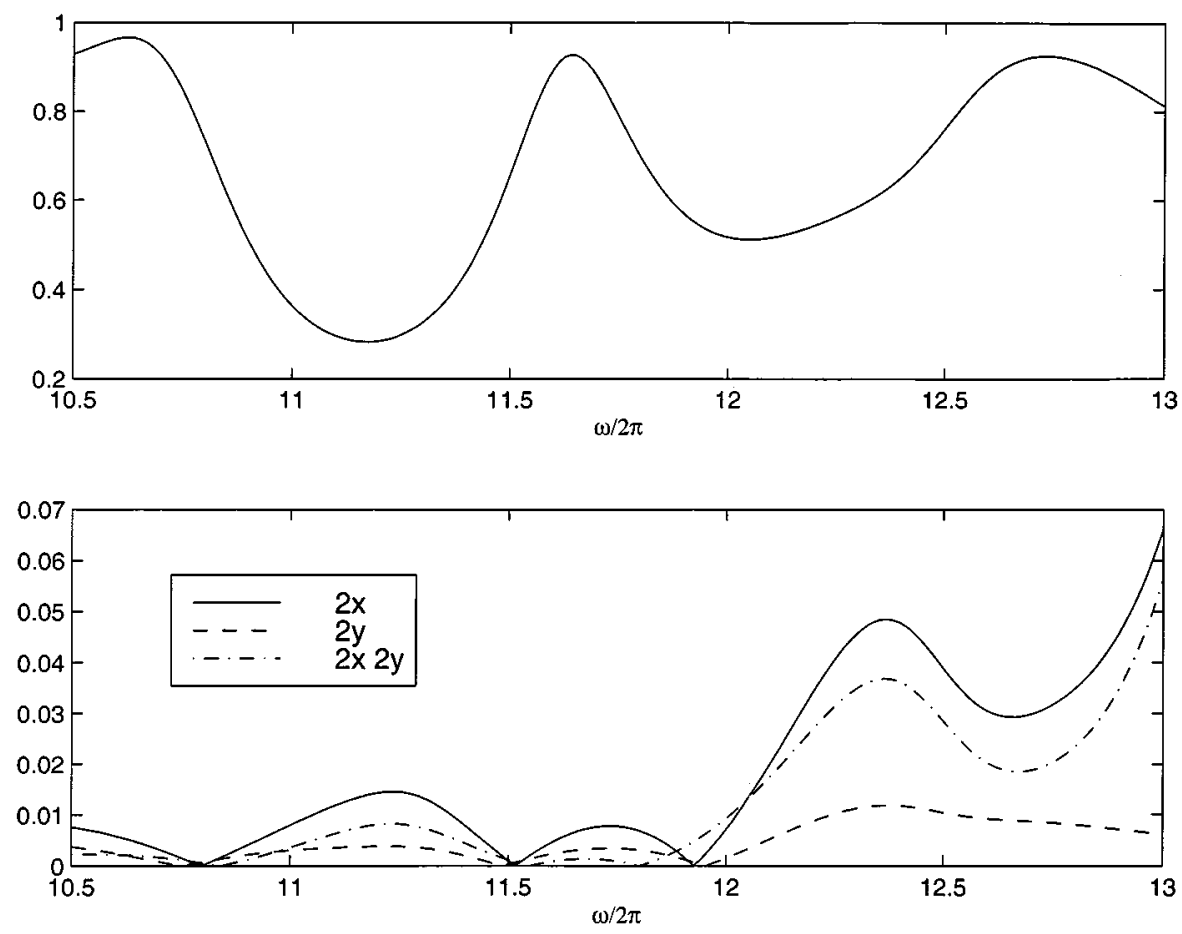

FIG. 6. Frequency response of test problem (37) (top). Relative error of the frequency response computed from the homogenized solution of the same test problem (bottom). Results shown for homogenization two levels in the $x$ direction, in the $y$ direction, and in both.

\subsection{One-Dimensional Models through Homogenization}

To reduce the complexity of solving the full Helmholtz equation (6), and to gain better understanding of the physical processes, one often wants to derive lower-dimensional models that capture the significant features of the full model. We could for instance try to find a model of the form

$$
\partial_{x}\left(c_{\mathrm{e}}(x, \omega)^{2} \partial_{x} u_{\mathrm{e}}\right)+\omega^{2} u_{\mathrm{e}}=0,
$$

where $u_{\mathrm{e}}$ is related to $u$, the solution to (6), in a simple way. One approach to this problem, usually called the effective index method $[4,20]$, is to link $c_{\mathrm{e}}(x, \omega)$ to the local width of the waveguide, denoted $d(x)$. For fixed $x$ and $\omega$ the coefficient $c_{\mathrm{e}}(x, \omega)$ is given as the speed of the lowest propagating mode in an infinite straight waveguide of (constant) width $d(x)$ at frequency $\omega$. This is a good approximation as long as $d$ varies slowly relative to the wavelength and the height itself, $\left|d_{x} \omega\right| \ll 1$ and $\left|d_{x} / d\right| \ll 1$. There are more sophisticated versions of this method, where the effective index is averaged with a weight function corresponding to the predicted energy of the solution, the weighted index method [1], and where a part of the solution is computed via Fourier transform, the spectral index method [19]. Another way to simplify (1) is to approximate it by the one-way wave equation, such that all energy is assumed to propagate in one direction. This is used in the beam propagation method [14], where the solution is computed by "time stepping" the equation in the $x$ direction. It gives accurate results when the variations in $c(x, y)$ are small. The main difference between these methods and the one we propose is that no specific physical 
considerations are required with our method. It can however, as we will see, provide physical interpretations of the simplified models.

We will here show how the homogenization technique described in Section 4 can be used to derive a family of one-dimensional models with different properties. The full twodimensional operator is first homogenized multiple levels, but only in the $y$ direction. It is subsequently truncated and identified with a discretization of a one-dimensional differential operator. The resulting operator and right-hand side will approximate a system of onedimensional partial differential equations with a size equaling the number of grid points left in the $y$ direction. The order of the differential operator corresponds to the bandwidth of the truncated operator. In conclusion, we have three different parameters that we can vary:

1. the size $n$ of the system of equations,

2. the order $r$ of the differential operator, and

3. the subspace $H_{0}$ used in the homogenization.

In general there is a trade-off between $n$ and $r$, such that a smaller $n$ requires a larger $r$. The reason is that, to maintain good accuracy, the bandwidth after truncation usually needs to increase with the number of levels that an operator is homogenized.

These one-dimensional models yield an approximation of the solution projected on a space spanned by only a few grid points in the $y$ direction. In view of the results in Section 5.2 this would still give a quantitative picture of the full solution. In particular, by using (40) it would be possible to compute a good approximation of the frequency response $H_{\text {pow }}$.

To exemplify the process described above, we will derive a one-dimensional model for the test problem given by

$$
\begin{aligned}
& w=1, \quad a=3 / 16, \quad S=10, \quad d=5 / 16, \quad b=1 / 8, \\
& \Lambda=1 / 8, \quad \tau=1 / 64, \quad \delta=1 / 64, \quad M=16, \quad N=64 \text {. }
\end{aligned}
$$

For this problem it is difficult to find a subspace where a scalar model $(n=1)$ is accurate without using high values of $r$. We will use a medium size model, with $n=4, r=2$. Numerical experiments with different wavelet systems suggest that in general the higher the order of the wavelets, the better the result. We show results when $H_{0}$ is given by the Daubechies wavelet system with four vanishing moments.

In Fig. 7 the frequency response for this problem is compared with the one given by the effective index method, described above. Since $d(x)$ has discontinuities the result is not good. In particular the resonance peak is at the wrong frequency. We shall see that our model fairs better. The new model is an approximation of the full problem after two levels of homogenization in the $y$ direction. The frequency response given by this homogenized problem and (40) is also plotted in Fig. 7. It agrees well with the full response, as expected.

Let us introduce the continuous $4 \times 4$ system model with the unknowns $u=\left(u_{1}, u_{2}, u_{3}\right.$, $\left.u_{4}\right) \in \mathbb{C}^{4}$ :

$$
\partial_{x}\left(B(x) \partial_{x} u\right)+C(x) u+\omega^{2} u=0, \quad B(x), C(x) \in \mathbb{R}^{4 \times 4} .
$$

This ansatz differs from (43) in that it also has a variable coefficient in the lower order term. However, none of the coefficients are assumed to be frequency dependent. This is preferable here, since it will reduce the cost of computing the frequency response of the filter.

We now proceed as follows. The discrete two-dimensional problem is homogenized two levels in the $y$ direction and afterwards the unknowns in the equation $\bar{L} \bar{u}=\bar{f}$ are reordered 


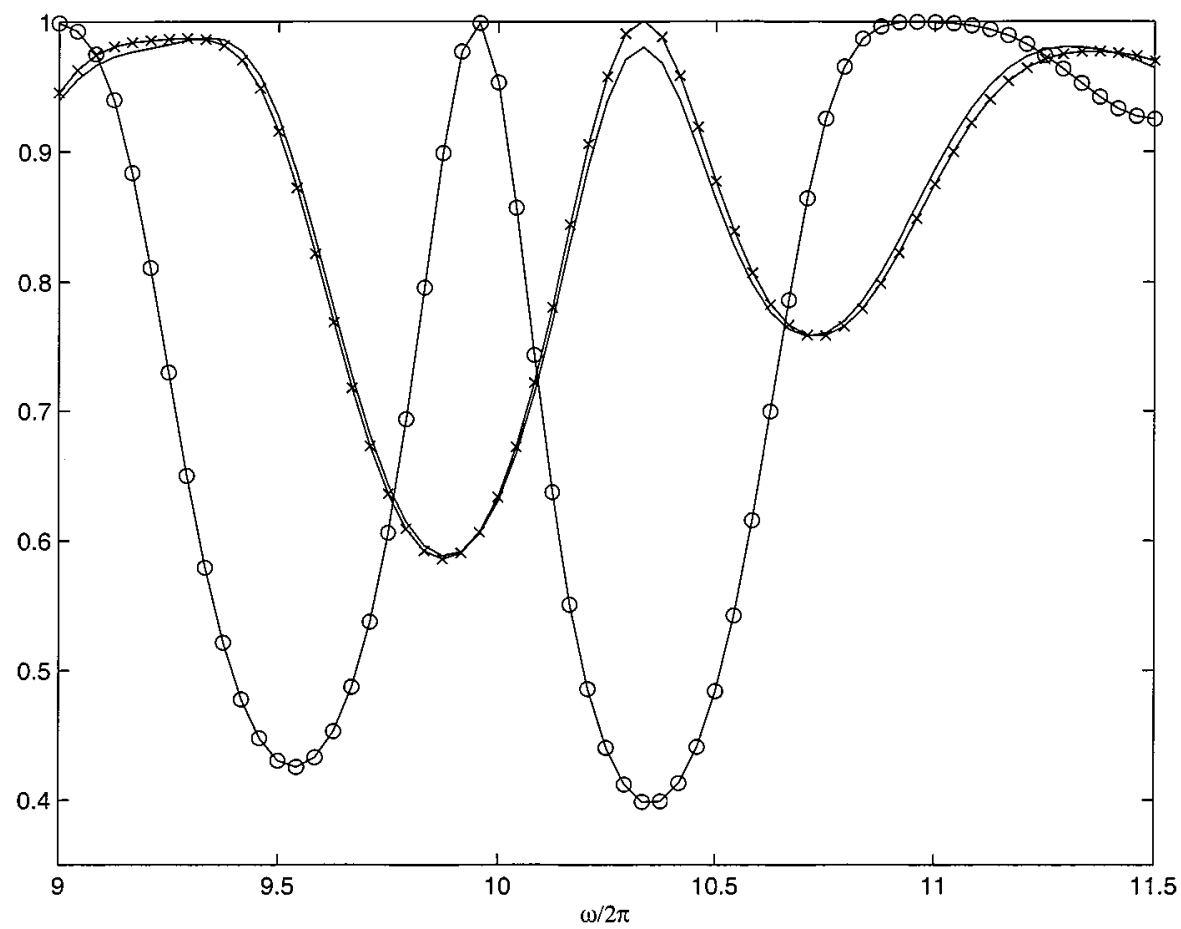

FIG. 7. Frequency response of test problem in (44) computed with the full two-dimensional model (solid line), computed after homogenization two levels in the $y$ direction of the full model (crosses), and computed with the effective index method (circles).

to the blocked form

$$
\left(\begin{array}{llll}
\bar{L}_{11} & \bar{L}_{12} & \bar{L}_{13} & \bar{L}_{14} \\
\bar{L}_{21} & \bar{L}_{22} & \bar{L}_{23} & \bar{L}_{24} \\
\bar{L}_{31} & \bar{L}_{32} & \bar{L}_{33} & \bar{L}_{34} \\
\bar{L}_{41} & \bar{L}_{42} & \bar{L}_{43} & \bar{L}_{44}
\end{array}\right)\left(\begin{array}{c}
\bar{u}_{1} \\
\bar{u}_{2} \\
\bar{u}_{3} \\
\bar{u}_{4}
\end{array}\right)=\left(\begin{array}{c}
\bar{f}_{1} \\
\bar{f}_{2} \\
\bar{f}_{3} \\
\bar{f}_{4}
\end{array}\right) .
$$

Each of the suboperators $\bar{L}_{i j}$ is truncated to tridiagonal form, which essentially corresponds to truncating the full operator $\bar{L}$ to 15 -diagonal form (before reordering). We identify the unknowns $\bar{u}_{j}$ and $\bar{f}_{j}$ with one-dimensional grid functions, $\bar{u}_{j}^{i} \approx u_{j}\left(x_{i}\right)$ and $\bar{f}_{j}^{i} \approx f_{j}\left(x_{i}\right)$ for some $f_{j}$. Furthermore, the truncated operators $\bar{L}_{i j}$ are identified with the discretization

$$
\frac{1}{h^{2}} \bar{L}_{i j}=\frac{1}{h^{2}} \Delta_{+} b_{i j}\left(x_{k-1 / 2}\right) \Delta_{-}+\bar{c}_{i j}\left(x_{k}\right)+\frac{1}{2} \gamma_{i j}\left(x_{k}\right)\left(\mathcal{E}+\mathcal{E}^{-1}\right)+\omega^{2}
$$

where $\mathcal{E}$ is the displacement operator defined by $\mathcal{E} \bar{u}^{k}=\bar{u}^{k+1}$. The $\gamma_{i j}$ terms are needed since $\bar{L}_{i j}$ is in general not symmetric. Each $\bar{L}_{i j}$ can be seen as a discretization of a second-order differential operator acting on the grid function $\bar{u}_{j}$. Assembling the suboperators by setting $B=\left\{b_{i j}\right\}, \tilde{C}=\left\{\tilde{c}_{i j}\right\}$, and $\Gamma=\left\{\gamma_{i j}\right\}$ we get

$$
\frac{1}{h^{2}} \Delta_{+} B\left(x_{k-1 / 2}\right) \Delta_{-} U^{k}+\tilde{C}\left(x_{k}\right) U^{k}+\frac{1}{2} \Gamma\left(x_{k}\right)\left(U^{k-1}+U^{k+1}\right)+\omega^{2} U^{k}=F^{k},
$$



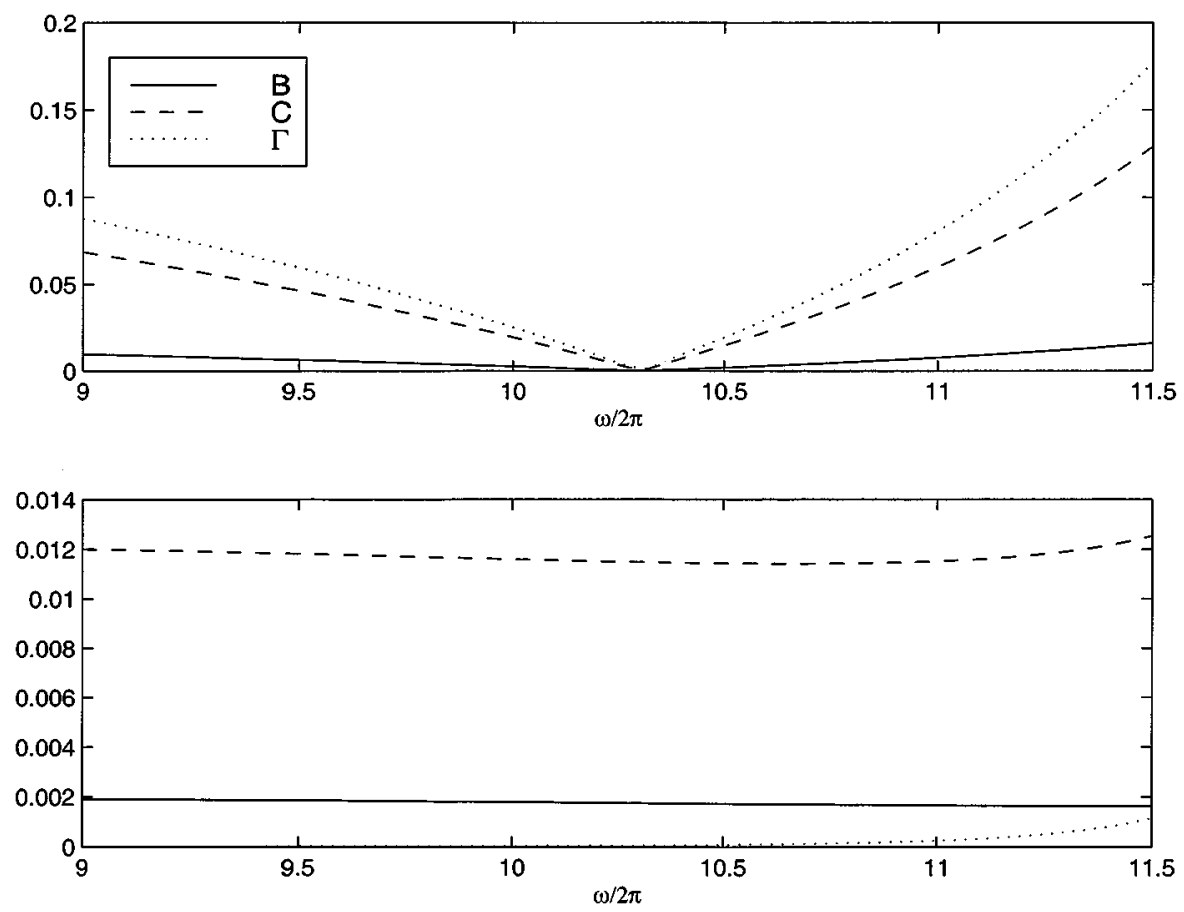

FIG. 8. Frequency dependency of coefficients. Relative $L_{2}$ difference between coefficients computed at $\omega$ and at $\omega / 2 \pi=10.3$ (top). Relative $L_{2}$ difference between imaginary and real part of coefficients (bottom).

where we also assembled the grid functions $U=\left\{\bar{u}_{j}\right\}$ and $F=\left\{\bar{f}_{j}\right\}$. If $C\left(x_{k}\right)=\tilde{C}\left(x_{k}\right)+$ $\Gamma\left(x_{k}\right)$ and $B\left(x_{k}\right)$ were real and independent of $\omega$ and if $F=0$ this equation could be identified with a second-order discretization of (45). Thus, the matrices $B(x)$ and $C(x)$ represent the effective material of the waveguide, similar to the $c_{\mathrm{e}}(x, \omega)$ that was computed with the effective index method.

In fact, the computed coefficients satisfy these requirements to a fairly good level of approximation, which is illustrated in Fig. 8. The coefficients are computed for a range of $\omega$, and the relative $L_{2}$ difference, compared to the coefficients computed at resonance, $\omega / 2 \pi=10.3$, is plotted in the top picture. The $B(x)$ coefficients vary on the order of only $1 \%$ in the interval. In the bottom picture the $L_{2}$-norm of the imaginary part of the coefficients relative to the real part is plotted, showing that this is at worst a little more than $1 \%$. This indicates that the assumption (45) is justified.

In Fig. 9 we show the frequency response computed in two different ways and compare them with the response for the two-dimensional model. The first way is to recompute the coefficients for each new frequency. This gives a very good agreement with the response of the full model. The second way is to compute all coefficients, once and for all, at the resonance frequency, $\omega / 2 \pi=10.3$. Even now the model captures the true response fairly well. We could hence calculate all parameters of the model for one single frequency and the model will remain approximately valid for the whole frequency band of interest.

The first and last rows of each $\bar{L}_{i j}$ correspond to boundary conditions. Therefore we have simply left the first and last two rows of each (truncated) $\bar{L}_{i j}$ intact in the methods above.

We also computed the frequency response after having set the right-hand side to zero and discarded the imaginary part of all coefficients (except the elements corresponding to 


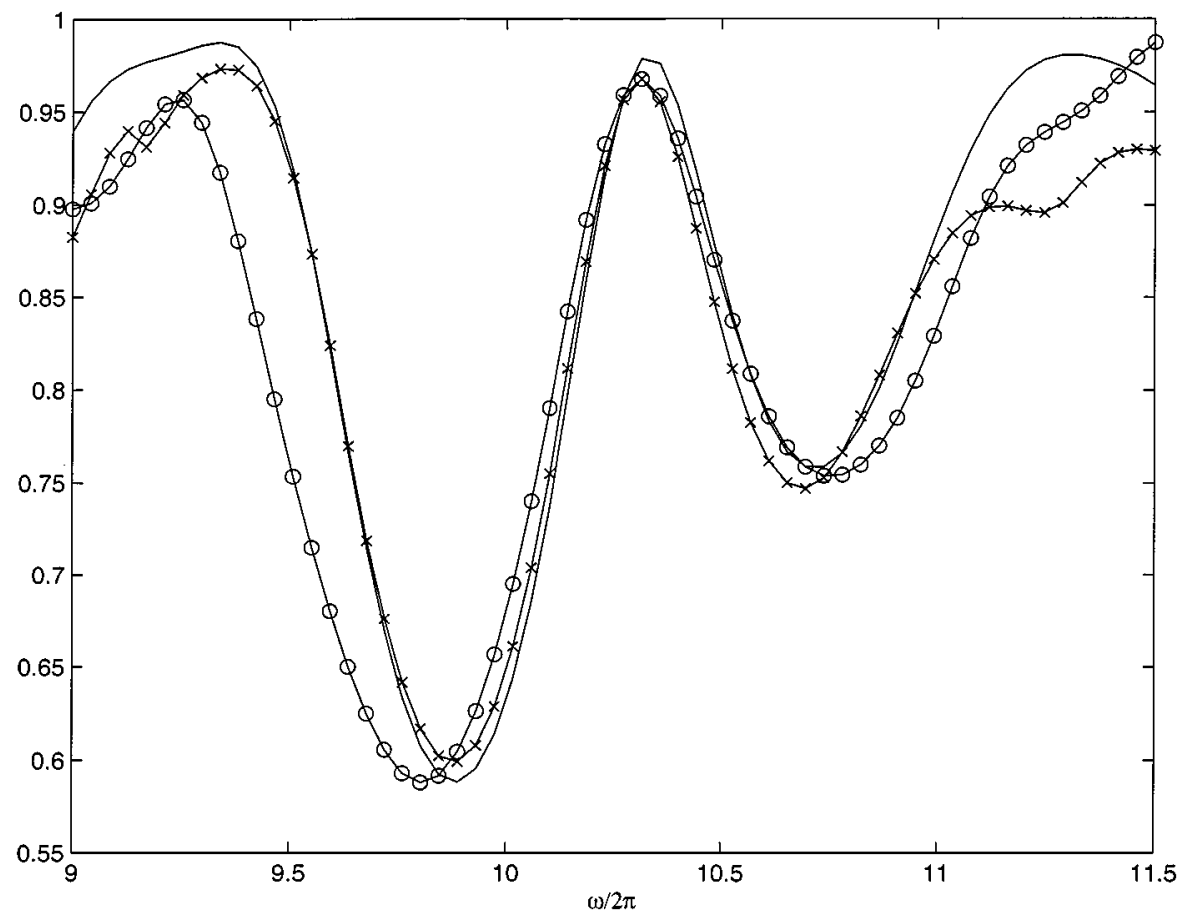

FIG. 9. Frequency response of test problem (44) computed using the one-dimensional model given in the text. Results shown when response was computed with the full two-dimensional model (solid line), with the onedimensional model and coefficients recomputed for each new frequency (crosses), and with the one-dimensional model and coefficients computed only once, for $\omega / 2 \pi=10.3$ (circles).

boundary conditions). This gave a slightly better response, but overall it did not alter the result in any significant way.

The computational gains can be large for the method described above. Since the bandwidth of the one-dimensional model is $n r$, the cost of computing the solution for $k$ frequencies is $C k n^{2} r^{2} N$ flops plus the $k$-independent cost of constructing the homogenized operator. At least for large $k$, the work of computing the solutions dominates. Comparing this with a direct method, which requires $C k N M^{3}$ flops, we get a cost ratio of

$$
\frac{n^{2} r^{2}}{M^{3}}
$$

which is often small. For the test problem (44) it equals $1 / 64$.

\subsection{The Homogenized Operator as a Subgrid Model}

One way to deal with the problem of small scales in a differential operator is to derive subgrid models that capture the effect of subgrid-scale phenomena on a coarse grid. There are many traditional ways to find such models in linear as well as nonlinear contexts. A number of methods are based on physical considerations for a specific application, for instance nonlinear turbulence models in computational fluid dynamics [25] and analytically derived local subcell models in computational electromagnetics [24]. Another example is the immersed interface method [18] used on Cartesian grids. In this method the subgrid variations of the shape of a material interface is taken into consideration by changing the 
updating stencils near the interfaces. Shock tracking or shock fitting can also be seen as subgrid models [2].

In this section we will use numerical homogenization to obtain linear subgrid models of the details of the waveguide and use them to solve a large problem on a coarse grid, as outlined in [3]. Our target problem is the same as in (37), only that we extend the waveguide by increasing the length $w$ and the number of gratings $S$. We use the same frequency as before, $\omega / 2 \pi=11$. To solve this problem on a coarse grid, we would need the homogenized operator and right-hand side. The key observation here is that a good approximation of these can be obtained directly from a much smaller problem, the analogue of the cell problem in classical homogenization.

In the waveguide example we identify four distinct parts of the problem: the initial part, a grating tooth, the quarter-wave step in the middle, and the end part. Let $\bar{u}^{\mathrm{i}}, \bar{u}^{\mathrm{m}}$, and $\bar{u}^{\mathrm{e}}$ denote the solution at grid points in the initial segment, middle segment, and end segment of the waveguide. Moreover, let $\bar{u}_{k}^{\mathrm{t}}$ be the solution at tooth $k$. Then we can decompose $\bar{L} \bar{u}=\bar{F}$ as

$$
(\bar{L}+\delta L)\left(\begin{array}{c}
\bar{u}^{\mathrm{i}} \\
\bar{u}_{1}^{\mathrm{t}} \\
\vdots \\
\bar{u}_{S / 2}^{\mathrm{t}} \\
\bar{u}^{\mathrm{m}} \\
\bar{u}_{S / 2+1}^{\mathrm{t}} \\
\vdots \\
\bar{u}_{S}^{\mathrm{t}} \\
\bar{u}^{\mathrm{e}}
\end{array}\right)=\left(\begin{array}{c}
\bar{F}^{\mathrm{i}} \\
\bar{F}_{1}^{\mathrm{t}} \\
\vdots \\
\bar{F}_{S / 2}^{\mathrm{t}} \\
\bar{F}^{\mathrm{m}} \\
\bar{F}_{S / 2+1}^{\mathrm{t}} \\
\vdots \\
\bar{F}_{S}^{\mathrm{t}} \\
\bar{F}^{\mathrm{e}}
\end{array}\right),
$$

where $\bar{F}$ follows the same partitioning as $\bar{u}$ and

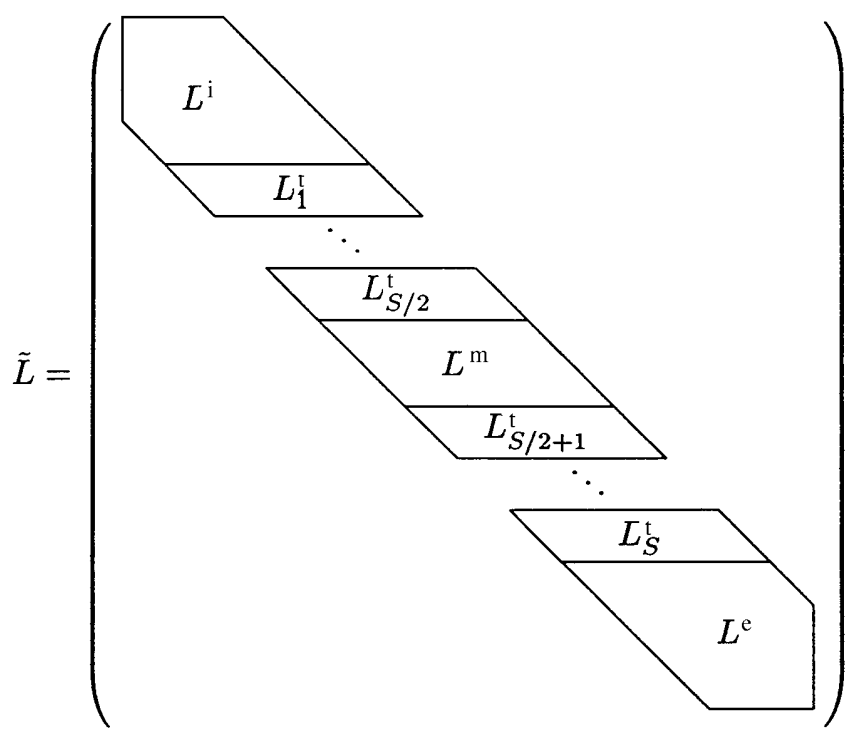

with elements being zero outside the delineated areas. The matrix $\delta L$ is the residual between $\bar{L}$ and $\bar{L}$, with all elements zero within the delineated areas. 
With this notation we now observe that since $\bar{L}$ is diagonal dominant, $\delta L$ will be small. Moreover,

$$
L_{k}^{\mathrm{t}} \approx L_{\ell}^{\mathrm{t}}, \quad \bar{F}_{k}^{\mathrm{t}} \approx \bar{F}_{\ell}^{\mathrm{t}}, \quad \forall k, \ell
$$

We will interpret $L^{\mathrm{i}}, L^{\mathrm{t}}, L^{\mathrm{m}}$, and $L^{\mathrm{e}}$ together with $\bar{F}^{\mathrm{i}}, \bar{F}^{\mathrm{t}}, \bar{F}^{\mathrm{m}}$, and $\bar{F}^{\mathrm{e}}$ as the subgrid models for the corresponding parts of the geometry. (Since $L_{k}^{\mathrm{t}}$ and $\bar{F}_{k}^{\mathrm{t}}$ are approximately the same for all $k$ we will henceforth drop the subindices.)

The strategy for constructing an approximation of the homogenized operator of the full problem is then clear. First, we compute an approximation of $L^{\mathrm{i}}, L^{\mathrm{t}}, L^{\mathrm{m}}$, and $L^{\mathrm{e}}$ by homogenizing a much smaller problem. At the same time we also obtain approximations of $\bar{F}^{\mathrm{i}}, \bar{F}^{\mathrm{t}}, \bar{F}^{\mathrm{m}}$, and $\bar{F}^{\mathrm{e}}$. Second, we assemble the subgrid models according to the block structures of (50) and (51) to obtain an approximation of $\bar{L}$ and $\bar{F}$. Finally, we neglect $\delta L$.

In our example we use (37) with $w=1$ and $S=10$ as our small problem. This is a well-resolved problem with eight gridpoints between the gratings and approximately 15 gridpoints per effective wavelength in the $x$ direction $(\beta / 2 \pi \approx 8.75$, with $\beta$ defined in Section 3.2). We homogenize the operator three levels in the $y$ direction and one level in the $x$ direction, corresponding to a grid size of $M=4$ and $N=64$. This gives a very coarse resolution in the $y$ direction, in particular no resolution of the gratings, and a reasonable resolution in the $x$ direction. This grid size should be enough to represent the solution in a quantitatively correct way.

For the large problems we denote the corresponding parameters with a prime sign. We will have $w^{\prime}>1$ and $S^{\prime}>10$ and we set $L^{\mathrm{i}^{\prime}}=L^{\mathrm{i}}, L^{\mathrm{m}^{\prime}}=L^{\mathrm{m}}, L^{\mathrm{e}^{\prime}}=L^{\mathrm{e}}$, and

$$
L_{k}^{\mathrm{t}^{\prime}}= \begin{cases}L_{k}^{\mathrm{t}}, & 1 \leq k \leq 2, \\ L_{3}^{\mathrm{t}}, & 3 \leq k \leq S^{\prime} / 2-2, \\ L_{k+\left(S-S^{\prime}\right) / 2}^{\mathrm{t}}, & S^{\prime} / 2-1 \leq k \leq S^{\prime} / 2+2, \\ L_{S / 2+3}^{\mathrm{t}}, & S^{\prime} / 2+3 \leq k \leq S^{\prime}-2, \\ L_{k+S-S^{\prime}}^{\mathrm{t}}, & S^{\prime}-1 \leq k \leq S^{\prime} .\end{cases}
$$

So far we have deliberately been vague about the exact size of the areas in (51) and hence the size of the subgrid models. We now introduce a parameter $v$, the bandwidth of $\bar{L}$, with which we can define all sizes at the same time. The value of $v$ will determine the accuracy of the solution. The results of this computation are shown in Fig. 10, where the relative $L_{2}$ error between the solution computed using the subgrid technique and the exact solution, projected onto the coarse subspace, is plotted as a function of $v$, for five different problem sizes. Our small problem ( $w=1, S=10$ ) is also included, for comparison, in which case $v$ just indicates the bandwidth of the truncated operator. Even for a problem 16 times as large as the small problem, the relative $L_{2}$ error can be reduced to $10 \%$, by choosing $v$ big enough. Note that even when the relative $L_{2}$ error is rather large, a good quantitative picture of the solution is obtained in the interior of the waveguide. This is exemplified for the case of $w=16$ and $v=22$ in Fig. 11, where the part of the solution with the largest errors is plotted. The relative $L_{2}$ error in this case is of the order of $50 \%$.

There are two different error sources for this technique. Let $\bar{L}_{\text {best }}^{\prime}$ be the approximation of $\bar{L}^{\prime}$ obtained by using the subgrid models without any truncation. Then the difference 


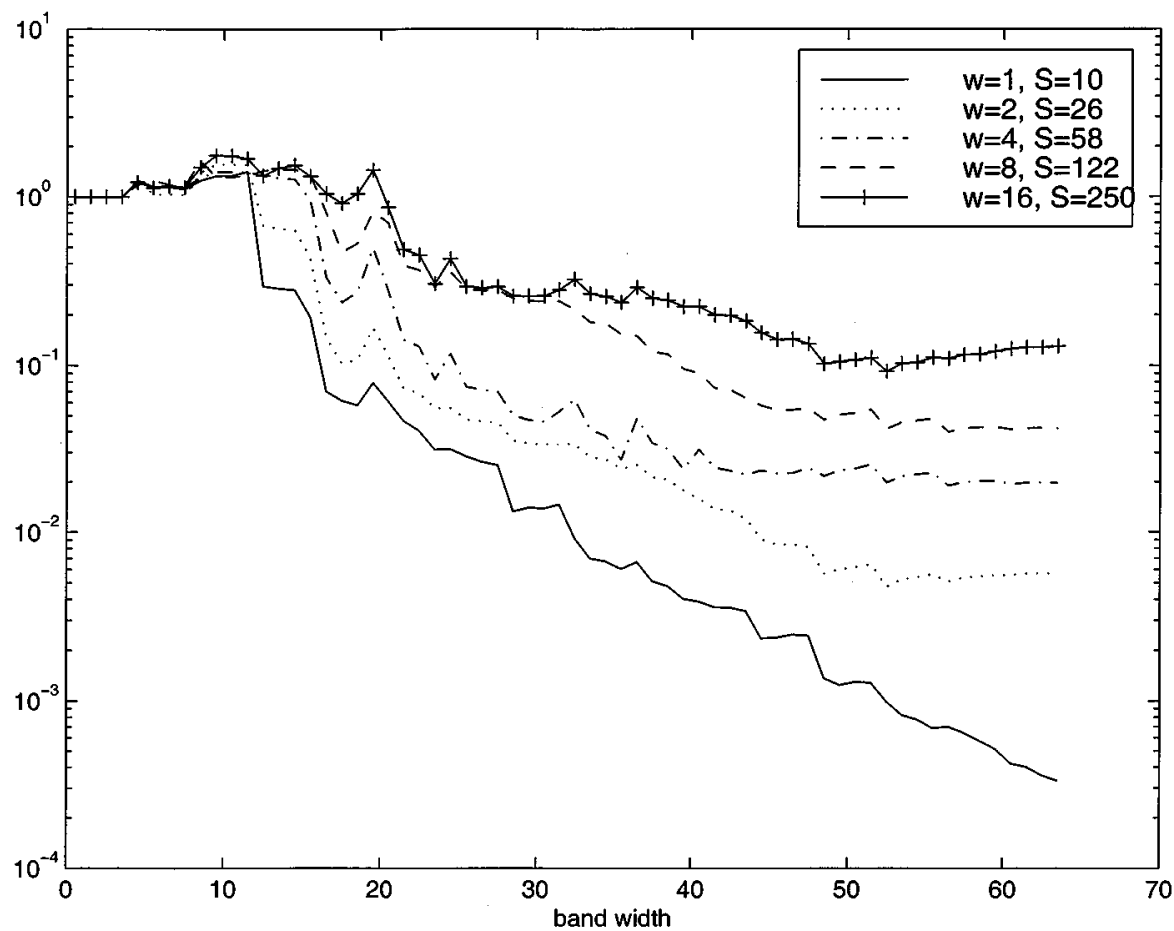

FIG. 10. Result of the subgrid technique. Relative $L_{2}$ error of solution as a function of the bandwidth.
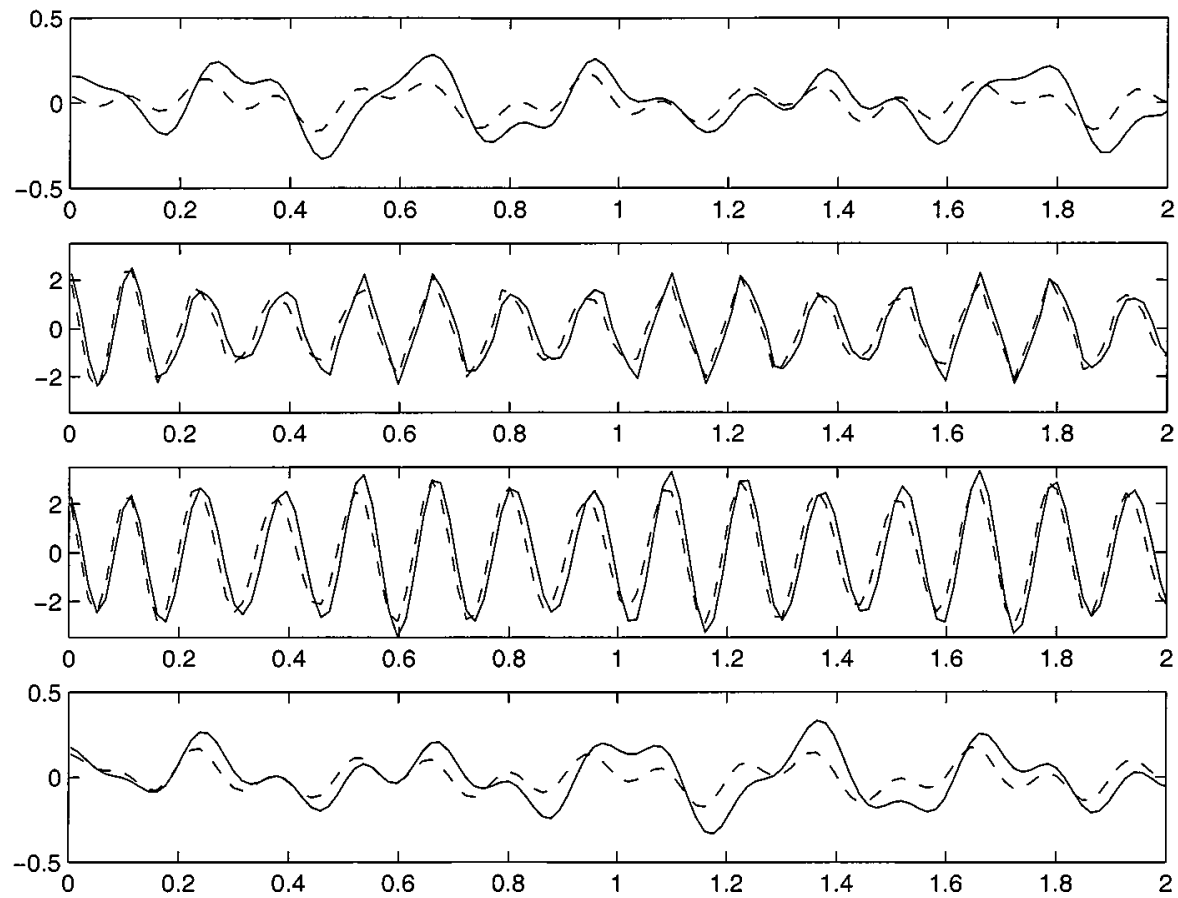

FIG. 11. Result of the large $w=16$ problem approximated with $v=22$. Plot of exact solution (solid) and approximate solution (dashed) as a function of $x$ for four $y$ values corresponding to the four grid points in the $y$ direction of the coarse mesh. Real part of $u$ shown in the $x$ interval [0,2], the region with the largest errors. 
between the exact homogenized operator $\bar{L}^{\prime}$ and the approximation, $\tilde{L}^{\prime}$, is

$$
\bar{L}^{\prime}-\tilde{L}^{\prime}=\bar{L}^{\prime}-\bar{L}_{\text {best }}^{\prime}+\bar{L}_{\text {best }}^{\prime}-\tilde{L}^{\prime} \equiv \delta L^{\prime}+\delta \tilde{L}^{\prime} .
$$

The error attributed to $\delta \tilde{L}^{\prime}$ will follow the same pattern as in Section 5.2 for truncated operators, while the error ascribed to $\delta L^{\prime}$ is independent of $v$. In Fig. 10 we see that all error curves, except the one for the small problem where $\delta L^{\prime}$ is zero, level out after a certain $v$ value. This point, $v^{*}$, is where the effect of $\delta L^{\prime}$ starts to dominate. From an accuracy point of view it is therefore meaningless to use $v>v^{*}$. In this case $v^{*} \approx 45$ independently of the problem size.

To estimate the gain in using the subgrid technique, let $M^{\prime}$ and $N^{\prime}$ denote the grid size of the large problem. The exact operator has a bandwidth of $M^{\prime}$. The cost to solve this problem is therefore $C N^{\prime} M^{\prime 3}$ flops with a direct solver. Suppose our coarse grid is of the size $N^{\prime} / 2^{n_{x}}$ and $M^{\prime} / 2^{n_{y}}$, corresponding to homogenization $n_{x}$ times in the $x$ direction and $n_{y}$ times in the $y$ direction. The direct solution using the subgrid model then costs $C N^{\prime} M^{\prime} v^{2} / 2^{n_{x}+n_{y}}$, giving a ratio between the costs of

$$
2^{-n_{x}-n_{y}}\left(\frac{v}{M^{\prime}}\right)^{2} .
$$

In our largest case, $w=16$, with $v=v^{*}=45$, we get an approximate ratio of $1 / 8$. We must also compute the subgrid model, although this only needs to be done once for all five computations above, and for the grid size of the small problem. This cost is dominated by the other factors.

\section{CONCLUSION}

We have showed how wavelet-based homogenization can be used to derive coarse-scale approximate models for an optical waveguide filter with gratings. The models accurately describe the original problem, because the method takes into account the effect of the finescale details. This has been demonstrated by a number of numerical tests. The method generalizes to other physical systems described by partial differential equations, when the approximate models are physically feasible.

The technique is promising for reducing the complexity of the problems, especially when several similar problems are to be solved. We have shown two examples of this: when the frequency response is computed and when several waveguides with different numbers of gratings are simulated.

The derived models can also provide additional insight into the physics of the problem. We have shown that the two-dimensional waveguide with gratings can be approximated by a one-dimensional $4 \times 4$ system of Helmholtz-type equations, with coefficients that vary spatially, but are independent of the frequency in a neighborhood of resonance. The coefficients in the derived operators can be interpreted as the effective material properties for the waveguide, and they can be studied to better understand the filter effect and the physical influence of the gratings. Similar studies can be made with the subgrid models, where simplified models can be suggested by the coefficients in the homogenized operator.

\section{ACKNOWLEDGMENT}




\section{REFERENCES}

1. M. J. Adams, P. C. Kendall, S. Ritchie, and M. J. Robertson, Theory for calculating approximate values for the propagation constants of an optical Rib waveguide by weighting the refractive indices, IEE Proc. A 134(8), 699 (1987).

2. J. Anderson, Computational Fluid Dynamics, The Basics with Applications (McGraw-Hill, New York, 1995).

3. U. Andersson, B. Engquist, G. Ledfelt, and O. Runborg, A contribution to wavelet-based subgrid modeling, Appl. Comput. Harmon. Anal. 7, 151 (1999).

4. T. M. Benson, R. J. Bozeat, and P. C. Kendall, Rigorous effective index method for semiconductor Rib waveguides, IEE Proc. J. 139(1), 67 (1992).

5. A. Bensoussan, J.-L. Lions, and G. Papanicolau, Asymptotic Analysis for Periodic Structures (North-Holland, Amsterdam, 1978).

6. G. Beylkin and M. Brewster, A multiresolution strategy for numerical homogenization, Appl. Comput. Harmon. Anal. 2, 327 (1995).

7. G. Beylkin, M. E. Brewster, and A. C. Gilbert, A multiresolution strategy for numerical homogenization of nonlinear ODEs, Appl. Comput. Harmon. Anal. 5, 450 (1998).

8. G. Beylkin, R. Coifman, and V. Rokhlin, Fast wavelet transforms and numerical algorithms I. Comm. Pure Appl. Math. 44, 141 (1991).

9. G. Beylkin and N. Coult, A multiresolution strategy for reduction of elliptic PDEs and eigen-value problems, Appl. Comput. Harmon. Anal. 5, 129 (1998).

10. G. Beylkin, J. Dunn, and D. L. Gines, LU factorization of non-standard forms and direct multiresolution solvers, Appl. Comput. Harmon. Anal. 5, 156 (1998).

11. I. Daubechies, Ten Lectures on Wavelets (Soc. for Industr. \& Appl. Math., Philadelphia, 1991).

12. M. Dorobantu and B. Engquist, Wavelet-based numerical homogenization, SIAM J. Numer. Anal. 35(2), 540 (1998).

13. L. Durlofsky, Numerical calculation of equivalent grid block permeability tensors for heterogeneous porous media, Water Resour. Res. 27, 699 (1991).

14. M. D. Feit and J. A. Fleck, Jr., Light propagation in graded-index optical fibers, Appl. Opt. 17(24), 3990 (1978).

15. A. C. Gilbert, A comparison of multiresolution and classical one-dimensional homogenization schemes, Appl. Comput. Harmon. Anal. 5(1), 1 (1998).

16. H. A. Haus and Y. Lai, Narrow-band optical channel-dropping filter, J. Lightwave Technol. 10(1), 57 (1992).

17. M. Levy, L. Eldata, R. Scarmozzino, R. M. Osgood, Jr., P. S. D. Lin, and F. Tong, Fabrication of narrowband channel-dropping filters, IEEE Photonics Technol. Lett. 4(12), 1378 (1992).

18. Z. Li, The Immersed Interface Method-A Numerical Approach for Partial Differential Equations with Interfaces, Ph.D. thesis (Department of Applied Mathematics, University of Washington, 1994).

19. P. W. A. McIlroy, Spectral index method: Single Rib waveguide, in Rib Waveguide Theory by the Spectral Index Method, edited by P. C. Kendall and P. N. Robson (Wiley, New York, 1990), pp. 70-122.

20. V. Ramaswamy, Strip-loaded film waveguide, Bell Syst. Tech. J. 53, 697 (1974).

21. O. Runborg, Multiscale and Multiphase Methods for Wave Propagation, Ph.D. thesis (Department of Numerical Analysis and Computing Science, KTH, Stockholm, 1998).

22. B. Z. Steinberg, J. J. McCoy, and M. Mirotznik, A multiresolution study of effective properties of complex electromagnetic systems, IEEE Trans. Antenn. Propag. 46(7), 971 (1998).

23. B. Z. Steinberg, J. J. McCoy, and M. Mirotznik, A multiresolution approach to homogenization and effective modal analysis of complex boundary value problems, SIAM J. Appl. Math. 60(3), 939 (2000).

24. A. Taflove, Computational Electromagnetics, The Finite-Difference Time-Domain Method (Artech House, Norwood, MA, 1995), Chapter 10.

25. D. Wilcox, Turbulence Modeling for CFD (DCW Industries, Inc., La Canada, California, 1993). 